child is under multidisciplinary team involving Paediatrics, ENT, Maxillofacial, Genetics and Speech and Language Therapy team.

Discussion VWS is dominantly inherited with prevalence of 1 in 100,000-200,000. However 30 to 50\% of cases arise as denovo mutations hence the diagnosis is made on a clinical basis. Congenital lip pits, cleft lip or palate with varying severity are common presentations in $70 \%$ of cases. Submucous cleft palate is a common feature, nevertheless is easily missed on physical examination. Hypernasal voice and bifid uvula are isolated in VWS. Hypodontia is a cardinal feature of VWS. Extra-oral manifestations are limb anomalies, popliteal webs and brain abnormalities. Accessory nipples, heart defects, and Hirschsprung's have also been reported.

Conclusion Overall affected parents carry a 50\% risk for each child, and more affected phenotypes gives rise to extreme effects in offspring. However, given more mild features are common, it is prudent to always be vigilant for cleft or lip abnormalities in all offspring. These children need multidisciplinary team approach and long-term follow up.

\section{GP44 ERYTHROPOIETIN AS A TREATMENT MODALITY IN HYPERHAEMOLYSIS COMPLICATING SICKLE CELL ANAEMIA}

Ana Louise Hawke*, Helena Conroy, Rosena Geoghegan, Corrina McMahon. Our Lady's Children's Hospital, Crumlin, Dublin, Ireland

\subsection{6/archdischild-2019-epa.110}

Introduction Hyperhaemolysis Syndrome (HS), a severe haemolytic transfusion reaction, is a rare complication in children with Sickle Cells anaemia (SCA) who require transfusion. Donor and autologous red cells are obliterated leading to a worsening of anaemia after transfusion. Erythropoietin has been reported as a treatment modality. This case series examines the experience in our tertiary Paediatric Haematology centre, of treating this haemolytic anaemia with Erythropoietin.

Design and Methods Patients were identified from the SCA patient database at Our Lady's Children's Hospital, Crumlin, Dublin in 2018.

Patient charts were reviewed Details of transfusions, presenting symptoms, examination findings, lab results and treatment modalities were recorded.

Results Three children being treated with erythropoietin following HS were identified.

Patient 1:

Diagnosed with SCA at birth. Transfusion programme started aged 3 years 6 months due to silent infarct on MRI brain.

Transfusion programme stopped aged 3 years 10 months as hyperhaemolysis suspected. Haemoglobin (Hob) fell post transfusion and $\mathrm{HbA}$ rose to $20 \%$ not $60-70 \%$ as would be expected. Autoantibodies were detected.

This was soon complicated by Acute Splenic sequestration with associated high fever. Hob at it's nadir 5.7 g/L. Erythropoetin commenced with good effect to date.

Patient 2:

SCA diagnosed aged 2 years. First transfusion aged 15 years due to crisis with Hob 6. 5 g/L. 5 weeks later admitted with abdominal pain and splenomegaly. Hob $7.45 \mathrm{~g} / \mathrm{L}$ and then fell to $5.7 \mathrm{~g} / \mathrm{L}$. Treated with Immunoglobulin(IVIG) as diagnosis of HS was suspected. Hob A-13.1\%. Commenced on erythropoietin and IV Iron.

2 alloantibodies detected. Hob maintained $>6 \mathrm{~g} / \mathrm{L}$ since then with Erythropoetin 3 times a week and oral iron replacement.

Patient 3:

SCA diagnosed aged 1 year. At age 4 years, 3 admissions with chest crises and associated anaemia.

Hob fell to a nadir of $4.9 \mathrm{~g} / \mathrm{L}$ post transfusion and episodes were treated with IVIG and IV methylprednisolone. Another chest crisis at 4 years 7 months precipitated trial of erythropoietin for 3 months with good effect. No further transfusions required. Erythropoetin restarted aged 6 years 6 months due to $\mathrm{Hob}$ of $5.7 \mathrm{~g} / \mathrm{L}$ in association with infection. Hob improved and patient's Hob now maintained $>6 \mathrm{~g} / \mathrm{L}$ on erythropoietin three times a week.

Conclusion HS is a rare complication of SCA which can cause significant worsening of anaemia that is difficult to treat. Erythropoetin can be used to maintain acceptable levels of haemoglobin to avoid transfusion.

\section{GP45 AUTOIMMUNE ENCEPHALITIS TRIGGERED BY HERPES SIMPLEX VIRUS 1 INFECTION}

${ }^{1}$ Ancuta Bilasco, ${ }^{2}$ Szidonia Florea*, ${ }^{3}$ Ramona Cirt, ${ }^{1}$ Anca Draganescu, ${ }^{1}$ Magda Vasile, ${ }^{1}$ Camelia Kouris, ${ }^{1}$ Cristina Negulescu, ${ }^{4,1}$ Monica Luminos. ${ }^{1}$ National Institute for Infectious Diseases 'Prof. Dr. Matei-Balş, Bucharest, Romania; ${ }^{2}$ Children's Emergency Hospital, ClujNapoca, Romania; ${ }^{3}$ Bucharest Oncology Institute 'Professor Doctor Alexandru Trestioreanu', Bucharest, Romania; ${ }^{4}$ 'Carol Davila' University of Medicine and Pharmacy, Bucharest, Romania

\subsection{6/archdischild-2019-epa.111}

Introduction Herpetic encephalitis is the most frequent central nervous system infection in children caused by viral etiology. It may be a trigger for anti-NMDA receptor (NMDAR) encephalitis with the onset of symptoms usually a few weeks after initial presentation with viral encephalitis.

Case report Approximately one third of the patients with herpetic encephalitis develop anti-NMDAR encephalitis. There are more hypotheses on how anti-NMDAR encephalitis may develop. First, herpes simplex virus 1 (HSV-1) infection causes an inflammatory destruction of neural tissue with subsequent release of neuronal antigens and formation of NMDAR antibodies; or, second, by molecular mimicry. Onset of symptoms is usually after 2-6 weeks after initial infection with HSV 1 . In children symptoms are usually represented by choreoathetosis and orofacial dyskinesias. The diagnosis is made by detecting NMDAR antibodies in cerebrospinal fluid (CSF).

We present the case of an 11 months male infant with no previous medical history, who presented with fever, anorexia, seizures with a focal onset and secondarily generalized, somnolence and progressive neurologic deterioration with onset of symptoms 6 days prior to admission. CSF studies showed a hypertensive, hemorrhagic fluid with low levels of glucose. HSV-1 was detected by real time PCR in CSF. Brain MRI revealed multiple cerebral lesions of different sizes predominantly affecting the right hemisphere, thalamus and basal ganglia with diffuse edema. EEG suggested diffuse cerebral dysfunction. Treatment with Acyclovir was started and continued for 28 days with notable decrease in 